\title{
Failure of Urine Dipsticks to Detect Ketosis in Rats
}

\author{
Maximilian Bielohuby Dominik Menhofer Barbara J.M. Stoehr Martin Bidlingmaier \\ Endocrine Research Unit, Medizinische Klinik - Innenstadt, Ludwig-Maximilians University, Munich, Germany
}

Among the dietary strategies to overcome adiposity and obesity, the omission of carbohydrates in low-carbohydrate, high-fat diets (LC-HFDs) has been proposed as a tool for weight loss [1]. Popular weight loss guidebooks, including the Atkins diet books [2], claim that the lack of carbohydrates in LC-HFDs always triggers ketosis which would contribute to the weight-reducing effects of these diets. However, this theory was widely criticized in literature. Apart from attempts to reduce body weight with LC-HFDs, ketogenic LC-HFDs are also used to intentionally induce ketosis in patients with epilepsy and lately also as a potential beneficial treatment in other neurological diseases such as Alzheimer's and Parkinson's disease [3]. Both indications to use LC-HFDs are subject to intense research including rodent models. Quantification of ketosis in these models often is a crucial part of the investigation. The method of choice to analyze the level of ketosis is measurement of $\beta$-hydroxybutyrate (HBA), acetone and acetoacetate, collectively known as ketone bodies. The gold standard to quantify ketone bodies is analysis of HBA in circulation and acetone in urine or breath. However, urine ketone dipstick tests are often used as a quick and noninvasive alternative to assess ketone body status in clinical practice. In humans, a large study in 21,000 healthy subjects demonstrated that analysis of urine by the dipstick method is a practical and sensitive screening tool also for detection of ketosis [4]. In rats, analysis of HBA and even breath acetone has been shown to be an accurate measure of mild to moderate systemic ketosis [5, 6]. However, many rodent studies use the urine dipstick method to analyze ketone body concentrations although, to the best of our knowledge, a systematic validation of this method has never been conducted in rodents. Likhodii and colleagues [6] measured ketone body concentrations in rat plasma (by an enzymatic assay) and rat urine (by the dipstick method). They state that the dipsticks failed to adequately predict the degree of systemic ketosis because they correlated only poorly with the levels of HBA in plasma.
Employing a nutritional rat model, we have recently reported that induction of ketosis with LC-HFDs is not only dependent on the absence of carbohydrates but also on low dietary protein [7]. In this study, we had analyzed HBA in serum and acetone in urine by laboratory methods because the dipstick ketone body measurements yielded inconsistent results. We now decided to systematically investigate suitability of the dipstick method to analyze ketone bodies in rat urine. 12-week old male Wistar rats ( $\mathrm{n}=15$; Harlan, Germany) were fed a ketogenic LC-HFD (Kliba Nafag; Provimi Kliba SA, Kaiseraugst, Switzerland). The composition of the diet was $92.8 \%$ fat, $5.5 \%$ protein and $1.7 \%$ carbohydrates (macronutrient specifications are given as a percentage of metabolizable energy). After 3 weeks on the diets, urine samples were collected by housing the rats individually without bedding on stable metal grids for $12 \mathrm{~h}$. Samples for each rat were taken from spontaneous 12-hour cumulative urine in addition to serum samples. Presence of ketone bodies in urine was first measured with commercially available dipsticks ('Ketostix', (Bayer, Leverkusen, Germany) and 'Ketodiabur Sticks' (Roche, Basel, Switzerland)) as by manufactures instructions. The color intensity of the dipsticks was compared with a color chart and transferred to the indicated numerical value. Urine samples were then stored at $-20{ }^{\circ} \mathrm{C}$ until quantification of acetone by headspace injection and subsequent GC/MS analysis (Laboratoriumsmedizin Dortmund, Dortmund, Germany). As a representative example, the figure shows the results of ketone body measurements in urine from 10 different rats. Laboratory measurement of acetone in urine and HBA in serum (data not shown) confirmed ketosis in all rats. Within the same samples, we found a large discrepancy between the results obtained from the dipsticks and the laboratory analysis. Interestingly, the dipstick method both underestimated and overestimated concentrations of ketone bodies in different rats. In some cases, the results from the dipsticks also varied considerably between the two manufacturers. Most strikingly, dipsticks from both companies did not reliably indicate presence of

\section{KARGER \\ Fax +497614520714 \\ Information@Karger.de}

www.karger.com (c) 2011 S. Karger GmbH, Freiburs

$1662-4025 / 11 / 0041-0081 \$ 38.00 / 0$

Accessible online at:

www.karger.com/ofa
Dr. med. Martin Bidlingmaie

Endocrine Research Unit, Medizinische Klinik - Innenstadt

University Hospital of the Ludwig-Maximilians University

Ziemssenstraße 1, 80336 Munich, Germany

Tel. +49 89 5160-2310, Fax -4457

Martin.Bidlingmaier@med.uni-muenchen.de 


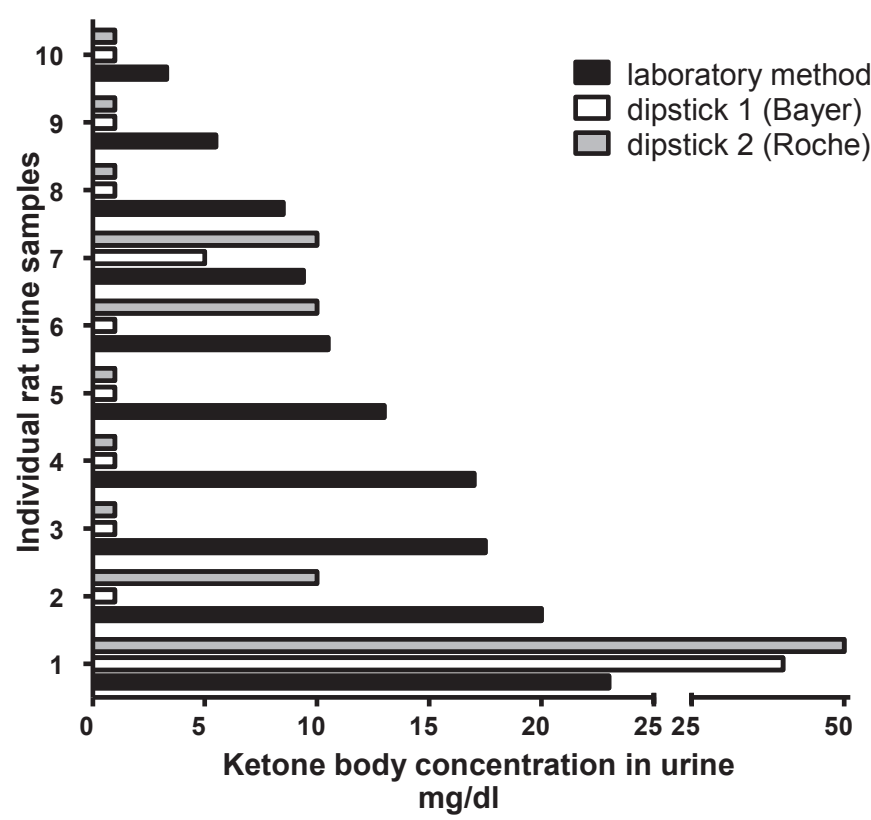

Fig. 1. Results of ketone body measurements in urine of 10 different rats fed a ketogenic LC-HF diet. Ketone body concentrations were analyzed by GC/MS-analysis (laboratory method, black bars) and by urine dipsticks from two different companies (dipstick 1, Bayer, white bars; dipstick 2, Roche, grey bars). Of note, negative dipstick results $(0 \mathrm{mg} / \mathrm{dl})$ were set to $1 \mathrm{mg} / \mathrm{dl}$ for better visualization in the figure. ketosis. In 12 out of the 15 samples analyzed, the laboratory analysis has shown acetone concentrations above $5 \mathrm{mg} / \mathrm{dl}$, but the dipsticks indicated that only 3 ('Ketostix') or 5 ('Ketodiabur Sticks') samples contain ketone bodies. In contrast to the poor correlation between laboratory and dipstick results of ketone body concentrations in urine, laboratory measurements of ketone body levels in urine (acetone) and serum (HBA) showed a significant correlation (Spearman $\mathrm{r}=0.557$; $\mathrm{p}<0.0001)$. Of course, the dipsticks used in this investigation (but also in many other rodent studies) are intended for usage in humans, and manufacturers do not claim that application of their dipsticks would yield reliable results in rats.

In conclusion, our data show that - although quick, easy and noninvasive - the urine dipstick methodology is not suitable to reliably detect and quantify ketosis in rats. We suggest that in rodents measurement of circulating HBA by laboratory methods reflects ketosis best, but laboratory analysis of acetone in urine might also be used as a less invasive technique to assess the level of ketosis in rats.

\section{Disclosure Statement}

All authors have nothing to disclose.

\section{References}

1 Shai I, Schwarzfuchs D, Henkin Y, Shahar DR, Witkow S, Greenberg I, et al: Weight loss with a low-carbohydrate, Mediterranean, or low-fat diet. N Engl J Med 2008;359:229-241.

2 Atkins R: Dr. Atkins New Diet Revolution. New York, Avon Books, 1992.

3 Waltz E: Epilepsy controlled by low-carb diet's effect on brain channels. Nat Med 2007;13:516-517.
4 Carel RS, Silverberg DS, Kaminsky R, Aviram A: Routine urinalysis (dipstick) findings in mass screening of healthy adults. Clin Chem 1987;33:2106-2108. 5 Likhodii SS, Musa K, Cunnane SC: Breath acetone as a measure of systemic ketosis assessed in a rat model of the ketogenic diet. Clin Chem 2002;48: $115-120$.
6 Likhodii SS, Musa K, Mendonca A, Dell C, Burnham WM, Cunnane SC: Dietary fat, ketosis, and seizure resistance in rats on the ketogenic diet. Epilepsia 2000;41:1400-1410.

7 Bielohuby M, Menhofer D, Kirchner H, Stoehr BJ, Muller TD, Stock P, et al: Induction of ketosis in rats fed low-carbohydrate, high fat diets depends on the relative abundance of dietary fat and protein. Am J Physiol Endocrinol Metab 2010; 300:E65-76. 